\title{
DIVERSIDADE E DISTRIBUIÇÃO DOS PORTUNIDEOS (CRUSTACEA, BRACHYURA) NO LITORAL DE ANCHIETA, ESPÍRITO SANTO, BRASIL
}

\author{
Renzo Gonçalves Tavares ${ }^{1}$ \\ Keltony de Aquino Ferreira ${ }^{2}$ \\ Erika Takagi Nunes ${ }^{3}$ \\ Adriane Araújo Braga ${ }^{4}$
}

Resumo: O objetivo deste estudo foi caracterizar a diversidade e a distribuição espaço temporal dos portunideos na região de Anchieta, litoral sul capixaba, relacionando com fatores ambientais como: temperatura, salinidade, profundidade, teor de matéria orgânica e textura do sedimento. Os indivíduos foram coletados no período de Março/2013 à Fevereiro/2015 em três pontos distintos. Não houve diferença significativa nos valores médios de temperatura, salinidade e teor de matéria orgânica. Foi coletado um total de 2.849 portunideos, compreendendo quatro espécies: Callinectes ornatus e Callinectes danae que foram consideradas constantes e Cronius ruber e Achelous spinimanus consideradas acessórias. A maior abundância de indivíduos foi registrada no $P 1$ por ser próximo ao estuário, região importante para o desenvolvimento e ciclo de vida desses animais, e com picos no outono para $C$. ornatus e na primavera para C. danae. A região de Anchieta pode ser considerada uma área importante para o estabelecimento e manutenção dessas espécies de portunideos.

Palavras-chave: Portunoidea; Siri; Índice de diversidade; Distribuição ecológica; Sul capixaba.

\footnotetext{
${ }^{1}$ Ciências Biológicas/Universidade Federal do Espírito Santo, Brasil. E-mail: renzo_tavares@hotmail.com.

${ }^{2}$ Ciências Biológicas/Universidade Federal do Espírito Santo, Brasil. E-mail: keltony.aquino@yahoo.com.br.

${ }^{3}$ Ciências Biológicas/Universidade Federal do Espírito Santo, Brasil. E-mail: erikatnunes@yahoo.com.br.

${ }^{4}$ Ciências Biológicas/Universidade Federal do Espírito Santo, Brasil. E-mail: dricrab@yahoo.com.br.
} 